\title{
Evaluation of the Food and Agriculture Organization's global school-based nutrition education initiative, Feeding Minds, Fighting Hunger (FMFH), in schools of Hyderabad, India
}

\author{
GM Subba Rao 1,*, D Raghunatha Rao ${ }^{1}, K_{\text {Kenkaiah }}^{2}$, Anil K Dube ${ }^{1}$ and KV Rameshwar \\ Sarma $^{1}$ \\ ${ }^{1}$ Extension \& Training Division, National Institute of Nutrition, Indian Council of Medical Research (ICMR), Jamai \\ Osmania PO, Hyderabad - 500007 AP, India: ${ }^{2}$ Division of Field Studies, National Institute of Nutrition, ICMR, \\ Hyderabad, India
}

Submitted 6 June 2005: Accepted 19 January 2006

\begin{abstract}
Objective: To assess the efficacy of the Food and Agriculture Organization's global school-based nutrition education initiative, Feeding Minds, Fighting Hunger (FMFH), in improving nutrition-related knowledge levels of schoolchildren.

Design: Adopting the cluster randomisation technique, five schools each in experimental and control groups were randomly chosen from the member schools of a voluntary organisation. Repeated measures of knowledge levels were carried out at three points in time to assess pre-, post-intervention and retention of knowledge gained during the intervention. Children from experimental schools were given nutrition education by teachers in the classroom setting using FMFH material.

Subjects/settings: Schoolchildren ( $n=358$ in the control group and $n=312$ in the experimental group) of grades VIII and IX from schools in Hyderabad, India.

Results: The classroom-based intervention resulted in a significant improvement $(P<0.01)$ in nutrition knowledge levels of schoolchildren in the experimental group. Significant improvement in knowledge was also observed in the control group. The effect size indicated that the improvement in knowledge levels of schoolchildren in experimental schools over control schools was medium $(d=0.40)$, indicating the efficacy of the FMFH programme in improving nutrition-related knowledge. No significant decrease $(P>0.05)$ in knowledge levels was observed after 2 months, indicating retention of the knowledge acquired through the intervention.

Conclusion: The FMFH programme provides an opportunity for schoolchildren to learn more about nutrition through their teachers in a classroom setting if the lesson plans are adapted to the local circumstances. Furthermore, it has the potential to make nutrition education interactive, effective and sustainable.
\end{abstract}

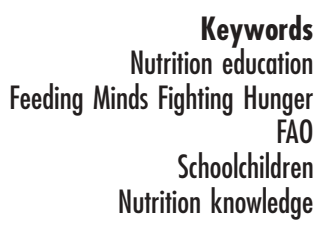

Nutrition education is a key element in promoting sustainable healthy eating behaviours and should start from early stages of life ${ }^{1}$. The importance of early learning of nutrition-related knowledge, attitudes and behaviours for future health is widely recognised ${ }^{2}$. The prevalence of malnutrition around the world, in the form of both undernutrition and overnutrition due to dietary excesses, is partly being attributed to low awareness.

In India alone, there are about 204 million undernourished people and more than $50 \%$ of children are suffering from undernutrition ${ }^{3,4}$. Undernutrition in the early stages of life can result in growth retardation, mental impairment and low immunity to disease in later life. On the other hand, overweight and obesity - especially in childhood and adolescence - are related to the burden of associated chronic diseases in adulthood ${ }^{5}$. With almost
20\% of Southeast Asia's population being constituted by adolescents, there is an urgent need to tackle this problem in India too ${ }^{6}$. Community trials suggest that nutrition education is an accessible and effective tool in developing healthy nutrition-related practices ${ }^{7}$. Schools provide the most effective and efficient way to reach a large segment of the population, including young people, their families and the community in general ${ }^{8}$. Hence, the development of innovative nutrition education curricula is a continuous and demanding process? .

Keeping this in mind, the Food and Agriculture Organization of the United Nations (FAO) along with a group of international and non-governmental organisations has launched a global education initiative called 'Feeding Minds, Fighting Hunger' (FMFH) with the aim of educating and motivating schoolchildren to get actively 
involved in creating a world free from hunger and malnutrition. In order to popularise this initiative in the Southeast Asian region, FAO and UNESCO (United Nations' Educational, Scientific and Cultural Organization) organised an inter-country workshop in August 2002 at the National Institute of Nutrition (NIN) in Hyderabad, India. As part of the country initiative, an exploratory study was carried out in Hyderabad to assess the efficacy of FMFH lesson plans in improving the knowledge levels of schoolchildren $^{10}$. The objectives of the study were: (1) to assess the current knowledge levels of middle-level schoolchildren and their science teachers on topics related to FMFH lessons; (2) to orient teachers on the concepts of FMFH and to educate middle-level schoolchildren through them; and (3) to evaluate the impact of school-based teaching of FMFH lessons on the improvement of nutrition-related knowledge levels of schoolchildren.

\section{Materials and methods}

\section{Study setting}

The study was conducted in schools of the old city of Hyderabad in association with a non-governmental organisation, the Confederation of Voluntary Associations (COVA). Out of 83 member schools of COVA, 49 schools whose medium of instruction was English were considered for the study. Informed consent was obtained from the heads of the participating schools and pupils.

\section{Sample size}

In the pilot study, a pre-tested knowledge assessment questionnaire (KAQ) consisting of 20 multiple-choice (closed-ended) questions was administered to 75 schoolchildren selected randomly from five schools. The 20 questions were related to hunger, food insecurity, nutrition, nutrition deficiency disorders and FAO; two additional questions related to their preferences in methods of learning were also included.

For the main study, the cluster randomisation procedure was used to determine the number of schools. The sample size was calculated from an expected improvement of 4 points (on a scale of 20) in mean scores after the intervention, with 95\% level of significance and 80\% power. From the total number of schools available, 10 schools were selected randomly and each school was again randomly allocated to either the control or the experimental group. Thus, five schools were treated as control group and the remaining five as experimental group. All children from grades VIII and IX, and the biological science teachers of these classes, were included in the study.

\section{FMFH lesson plans}

Three different lesson plans of FMFH are suggested for Primary, Intermediate and Secondary school levels. Each lesson contains background information for the teacher, objectives, concepts and contents to be covered in the classroom during implementation. Three common lesson plans for all school levels deal with three different topics: (1) What are hunger and malnutrition and who are hungry? (2) Why are people hungry and malnourished? (3) What can we do to help end hunger? Apart from these guidelines, a variety of classroom activities including teaching aids and discussion points are also provided. For the purpose of the study, only the intermediate level lesson plan was used to educate the students in the experimental schools.

\section{Assessment of knowledge levels of schoolchildren}

Baseline data of schoolchildren from the control group $(n=358)$, the experimental group $(n=312)$ and their teachers $(5+5)$ were collected by administering the same KAQ that was used in the pilot study.

\section{Statistical analyses}

Data from the KAQ were analysed using the SPSS package version 11.5 (SPSS Inc., Chicago, IL, USA). Significant improvement in the knowledge levels of schoolchildren was observed even in the control group in an earlier study conducted in schools of Hyderabad ${ }^{11}$. Therefore, in the present study, the effect size was used to assess extent of the intervention's effect in improving knowledge levels in the experimental schools over the control schools?

\section{Intervention}

Teacher training workshops

A teacher training workshop was organised in March 2003 for the biological science teachers of grades VIII and IX of the experimental schools. The teachers were trained in the concepts of FMFH and different strategies of communication, in order to modify the FMFH lesson plans to suit the local needs of their schoolchildren. A follow-up workshop was conducted in July 2003 to reinforce the knowledge acquired in the previous workshop before the teachers implemented the FMFH lesson plans in their respective schools.

\section{Development of communication materials}

The following communication materials were developed based on the preferences indicated by the students in the pilot study, in consultation with the teachers:

- Posters: One poster on functions of foods and three posters on micronutrient deficiency disorders anaemia, vitamin A and iodine deficiency disorders were identified from existing posters of the NIN and modified. In addition, posters relating a Hunger Map of Asia, a Hunger Map of the World, vulnerable groups, food systems and what can children do to help end hunger and malnutrition were adapted from the FMFH lesson plans. These topics were identified with the help 
of teachers during the first teacher training workshop. Each school was given a complete set of 10 posters.

- Skit: A skit covering all the concepts mentioned in FMFH lesson plans was developed and performed using the children's theatre group of COVA to reinforce classroom education.

Implementation of FMFH lesson plans

In the experimental schools, teachers implemented FMFH lesson plans between August and October 2003 using the communication materials along with various classroom activities.

\section{Post-intervention knowledge assessment}

The post-intervention knowledge assessment was carried out by administering the same KAQ that was used at baseline to 254 students in the control group and 216 students in the experimental schools. The children were instructed not to discuss among themselves while answering the questionnaire. One school opted out of the study and some children were not present at the time of administration of the post-intervention questionnaire. As there was no significant difference in mean scores between experimental schools at baseline, the dropout did not affect the overall outcome.

Furthermore, retention of the concepts of FMFH lesson plans was also studied by administering the same questionnaire after a gap of 2 months for the experimental group. For the purpose of analysis, each right answer was assigned a score of one and the wrong answer was scored zero.

\section{Results}

\section{Pilot study}

The mean score ( \pm standard error, SE) of 75 children of all five schools was $8.36 \pm 0.36$. About $80 \%$ of the schoolchildren preferred to learn through classroom lectures, followed by teaching aids (such as charts and posters) and role play.

\section{Main study}

Mean score $( \pm$ SE) on the concepts of FMFH lesson plans of biological science teachers was $14.20 \pm 0.66$ in the control schools and $13.00 \pm 1.29$ in the experimental schools at baseline. However, an increase in knowledge levels among the biological science teachers of the experimental schools $(17.50 \pm 0.64)$ was observed after the intervention.

Baseline scores of schoolchildren in the control and experimental schools showed that there was no significant difference $(t=1.43 ; P=0.2)$ between the groups, indicating homogeneity in the groups (Table 1 ).

Post-intervention results indicated a significant improvement $(t=12.72 ; P=0.000)$ in the knowledge
Table 1 Knowledge levels of the schoolchildren at baseline

\begin{tabular}{lccc}
\hline Group & $n$ & $\begin{array}{c}\text { Knowledge score } \\
(\text { mean } \pm \text { SE) }\end{array}$ & Significance \\
\hline Control & 358 & $8.66 \pm 0.12$ & $t=1.43 ; P=0.2(\mathrm{NS})$ \\
Experimental & 312 & $8.95 \pm 0.17$ & \\
\hline SE - standard error; NS - not significant.
\end{tabular}

levels of the experimental group (Fig. 1). Significant improvement ( $t=7.95 ; P=0.000$ ) was also observed in the knowledge levels of the control group (Fig. 1). However, comparisons between the mean improvement in knowledge levels of the control $(1.65 \pm 0.21( \pm \mathrm{SE}))$ and experimental groups $(3.09 \pm 0.19( \pm \mathrm{SE}))$ revealed a significant increment $(t=4.54 ; P=0.000)$ in the experimental group versus the control group, indicating the efficacy of the intervention (Fig. 2). Regarding the retention of knowledge gained during the intervention, comparison of post-intervention-1 and post-intervention-2 mean scores of schoolchildren in the experimental group showed no significant difference $(t=1.7 ; \quad P=0.09)$ (Fig. 1), indicating that there was retention of knowledge.

\section{Effect size}

The effect size is the average percentile standing of the average treated (or experimental) group relative to the average untreated (or control) group. In the present study, the effect size of the difference in improvement in nutrition knowledge after the intervention between experimental and control groups was $d=0.40$, indicating that the mean of the treated group is at the 66th percentile of the untreated group.

Effect size can also be interpreted in terms of the percentage of non-overlap of the treated group's scores with those of the untreated group. In the present study, the effect size of 0.40 indicated a non-overlap of $27.4 \%$ in the two distributions, establishing that the intervention's effect was of medium magnitude as per Cohen's standard ${ }^{12}$.

\section{Discussion}

Nutrition education at a young age influences the attitudes of schoolchildren and enhances the knowledge and skills required for them to understand contemporary food and nutrition issues ${ }^{13}$. In the present investigation, teaching of FMFH material adapted by the biological science teachers in the experimental group resulted in a significant increase in the knowledge level of the schoolchildren. Similar studies by Raghunatha Rao et al. showed that an intervention comprising nutrition education through classroom teaching in experimental schools significantly improved the knowledge levels of adolescent school-going girls in an urban slum ${ }^{14}$. Significant improvement in knowledge levels was additionally observed in their control group, 


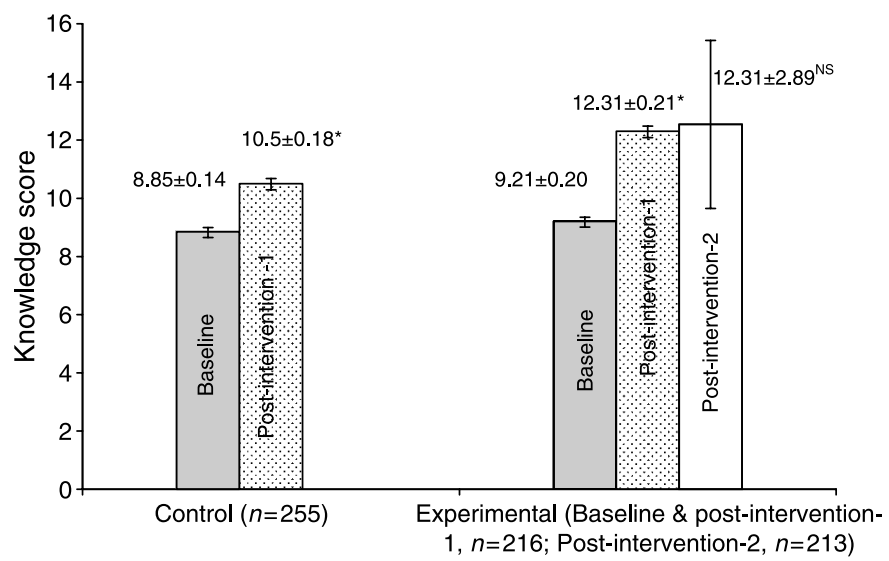

Fig. 1 Comparison of knowledge scores of control and experimental groups at baseline and after the intervention; values are means with standard error shown by vertical bars. ${ }^{*}$ Mean scores were significantly different compared with baseline: $P=0.000(t=7.95$ in control group, $t=12.72$ in experimental group). NS - not significant, $P=0.09(t=1.7)$ between post-intervention-1 and post-intervention-2

as also reported in the present study. The significant post-intervention improvement in knowledge levels of the students in control schools could be attributed to other factors such as the nutrition lessons in their curriculum, exposure to various media and the influence of parents, teachers and peer groups ${ }^{15}$. However, the significant improvement in nutrition knowledge of the experimental group through classroom education using FMFH lesson plans over the control group was medium, as shown by the effect size (Cohen's $d$ ). In a similar study using a computer-based nutrition education tool, Kreisel reported no detectable difference $(d=0.134)$ in nutrition knowledge between control and intervention schools and interpreted that the results were due to study limitations 9 . Slavin indicated that the impact of educational programmes could be considered successful when the effect size is 0.2 or greater $^{16}$. The effect size was medium $(d=0.40)$ in the present study, indicating that the FMFH programme is effective, efficacious and can be implemented in a classroom setting.

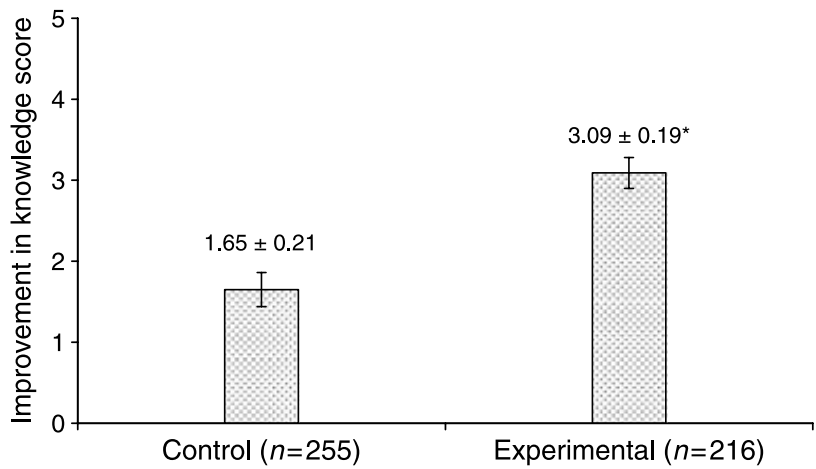

Fig. 2 Improvement in knowledge scores of the schoolchildren after the intervention; values are means with standard error shown by vertical bars. *Mean improvement was significantly different compared with the control group: $P=0.000(t=4.54)$

\section{Acknowledgements}

The investigators are thankful to the Indian Council of Medical Research (ICMR) for funding the study; Dr B Sivakumar, then Director of NIN, for his encouragement; and Mr Ajay Kumar, Schools Co-ordinator, COVA, for his support. Thanks are also due to the management teams and teachers of all schools where the study was conducted. We also thank the members of the Scientific Advisory Committee, NIN for their guidance and suggestions. The help rendered by colleagues at NIN is gratefully acknowledged.

Contributors: G.M.S.R. (Principal Investigator) participated in developing the study protocol, securing the funds, developing the KAQ and communication tools; D.R.R. (Co-Investigator and Guarantor) assisted in developing the KAQ, administration of the KAQ and preparation of the manuscript; K.V. (Co-Investigator) assisted in designing the study and performed the statistical analyses; A.K.D. (Co-Investigator) assisted in conducting the teacher training workshops; K.V.R.S. (Co-Investigator), being the head of the Extension \& Training Division, provided overall guidance for the study.

Conflict of interest: None stated.

\section{References}

1 Foerster SB, Heimendinger J, DiSogra LK, Pivonka E. The National 5 A Day for Better Health! Program: an American nutrition and cancer prevention initiative. In: Wheelock V, ed. Implementing Dietary Guidelines for Healthy Eating. London: Chapman \& Hall, 1997; 447-79.

2 Tones K, Tilford S. Health education in schools. Health Education: Effectiveness, Efficiency and Equity. London: Chapman \& Hall, 1994; 121.

3 Krishnaswami K. Country profile: India. Nutritional disorders - old and changing. Lancet 1998; 351(9111): 1268-9.

4 Food and Agriculture Organization (FAO). Feeding Minds, Fighting Hunger - A World Free from Hunger and Malnutrition. Rome: Publishing and Multimedia Service, Information Division, FAO, 2001; 5-8. 
5 Narayan KM, Campagna AF, Imperatore G. Type 2 diabetes in children: a problem lurking for India? Indian Pediatrics 2001; 38(7): 701-4.

6 Griffiths PL, Bentley ME. The nutrition transition is underway in India. Journal of Nutrition 2001; 131(10): 2692-700.

7 Kelder SH, Perry CL, Lytle LA, Klepp KI. Community-wide youth nutrition education: long-term outcomes of the Minnesota Heart Health Programme. Health Education Research 1995; 10(2): 119-31.

8 Perez-Rodrigo C, Javier A. School-based nutrition education: lessons learned and new perspectives. Public Health Nutrition 2001; 4(1A): 131-9.

9 Kreisel K. Evaluation of a computer-based nutrition education tool. Public Health Nutrition 2004; 7(2): 271-7.

10 Food and Agriculture Organization (FAO). Report on FAO-UNESCO Inter-Country Workshop on Feeding Minds, Fighting Hunger and Malnutrition beld at NIN, India, 27-29 August 2002. Hyderabad: National Institute of Nutrition, 2002
11 Vijayapushpam T, Menon KK, Raghunatha Rao D, Anthony GM. A qualitative assessment of nutrition knowledge levels and dietary intake of schoolchildren in Hyderabad. Public Health Nutrition 2003; 6(7): 683-8.

12 Cohen J. Statistical Power Analysis for the Behavioral Sciences, 2nd ed. Hillsdale, NJ: Lawrence Erlbaum Associates, 1988; 21-5.

13 Lytle LA. Nutrition education for school-aged children Journal of Nutrition Education 1995; 27(6): 298-313.

14 Raghunatha Rao D, Vijayapushpam T, Anthony GM, Subba Rao GM, Rameshwar Sarma KV. Nutrition knowledge and dietary habits of school going adolescent girls in Hyderabad. In: Proceedings of National Conference on Human Health and Nutrition: A Biotechnological Approach. Mumbai: VN Bandodkar College, 2004; 84-9.

15 Clancy-Hepburn K, Hickey AA, Nevill G. Children's behaviour responses to TV food advertisements. Journal of Nutrition Education 1974; 6: 93.

16 Slavin RE. A reader's guide to scientifically based research. Educational Leadership 2003; 60: 12-16. 